Draft VERSION OCTOBER 4, 2018

Typeset using LATEX twocolumn style in AASTeX61

\title{
THE PHYSICAL NATURE OF SUBDWARF A STARS: WHITE DWARF IMPOSTORS
}

\author{
Warren R. Brown, ${ }^{1}$ Mukremin Kilic, ${ }^{2}$ And A. Gianninas ${ }^{2}$ \\ ${ }^{1}$ Smithsonian Astrophysical Observatory, 60 Garden St, Cambridge, MA 02138 USA \\ ${ }^{2}$ Homer L. Dodge Department of Physics and Astronomy, University of Oklahoma, 440 W. Brooks St., Norman, OK, 73019 USA
}

(Accepted March 17, 2017)

\begin{abstract}
We address the physical nature of subdwarf A-type (sdA) stars and their possible link to extremely low mass (ELM) white dwarfs (WDs). The two classes of objects are confused in low-resolution spectroscopy. However, colors and proper motions indicate that sdA stars are cooler and more luminous, and thus larger in radius, than published ELM WDs. We demonstrate that surface gravities derived from pure hydrogen models suffer a systematic $\sim 1$ dex error for sdA stars, likely explained by metal line blanketing below $9000 \mathrm{~K}$. A detailed study of five eclipsing binaries with radial velocity orbital solutions and infrared excess establishes that these sdA stars are metal-poor $\simeq 1.2 M_{\odot}$ main sequence stars with $\simeq 0.8 M_{\odot}$ companions. While WDs must exist at sdA temperatures, only $\sim 1 \%$ of a magnitude-limited sdA sample should be ELM WDs. We conclude that the majority of sdA stars are metal-poor A-F type stars in the halo, and that recently discovered pulsating ELM WD-like stars with no obvious radial velocity variations may be SX Phe variables, not pulsating WDs.
\end{abstract}

Keywords: binaries: close — binaries: eclipsing — Galaxy: stellar content — stars: atmospheres — white dwarfs 


\section{INTRODUCTION}

Since the pioneering work of Payne (1925), astronomers have deduced the physical properties of stars through the combination of spectroscopy and stellar atmosphere models. Different spectral features are sensitive to different stellar atmosphere parameters. The modern approach is to compare an observed stellar spectrum to a grid of synthetic spectra calculated over a wide range of parameters. The most important parameters are effective temperature $T_{\text {eff }}$, surface gravity $\log g$, and metal abundance $[\mathrm{M} / \mathrm{H}]$. Once the stellar parameters are constrained, a comparison with evolutionary tracks yields a physical interpretation of the star. Consider a spectral A-type, $T_{\text {eff }}=8000 \mathrm{~K}$ star, which is the focus of this paper. If the star has $\log g=8$, it is a normal hydrogen-atmosphere WD; if the star has $\log g=4$, it is a normal main sequence A-type star. But if the star has $\log g=6$, it is something else.

In 2010 we began the ELM Survey, a spectroscopic survey targeting extremely low mass (ELM) WD candidates in the range $5 \lesssim \log g \lesssim 7$ and $8000 \mathrm{~K} \lesssim T_{\text {eff }} \lesssim$ 22,000 K (Kilic et al. 2010; Brown et al. 2010). ELM WDs are interesting because they form in ultra-compact binaries; the Universe is not old enough to make them through single-star evolution (Webbink 1984; Iben 1990; Marsh et al. 1995). Indeed, we have found and published 76 ELM WDs in short-period binaries over the past seven years (Kilic et al. 2011, 2012; Brown et al. 2012, 2013, 2016a; Gianninas et al. 2015). The median binary orbital period is 6 hrs, which means that half of the observed sample will merge within a Hubble time. The merger rate implies that most ELM WD binaries will not undergo stable mass transfer or explode as supernovae, but will undergo unstable mass transfer and merge into single massive WDs (Brown et al. 2016b).

A recent analysis of Sloan Digital Sky Survey (SDSS) spectra has complicated the picture of ELM WDs. Kepler et al. $(2015,2016)$ fit pure hydrogen and pure helium atmosphere models to the spectra of candidate WDs in SDSS, and find thousands of $\log g \sim 6$ objects at $T_{\text {eff }}<9000 \mathrm{~K}$ temperatures. The number of these $\log g$ $\sim 6$ objects is too large, by two orders-of-magnitude, to be explained as the cooler cousins of ELM WDs found in the ELM Survey. Yet their surface gravities are too great to be explained as metal-poor main sequence stars. Arguments both for and against the ELM WD interpretation appear in conference proceedings (Pelisoli et al. 2017; Hermes et al. 2017). Kepler and collaborators refer to these objects as subdwarf A (sdA) spectral-type stars, and their physical interpretation is unclear.

Perhaps sdA stars are thermally bloated WDs, like the class of EL CVn binaries discovered by Maxted et al.
(2013, 2014). However thermally bloated WDs, which are believed to have recently had their envelopes stripped by companions, are 10 to 100 times lower in luminosity, and 3 to 10 times smaller in radius, than their more massive companions (e.g., Carter et al. 2011; Maxted et al. 2014; Rappaport et al. 2015). sdA stars differ because they are the primaries: sdA stars dominate the light of their systems, and sdA stars in the eclipsing binaries presented here have comparable radii to their companions.

In this paper we address two questions at the base of the sdA mystery. 1) Are we measuring $T_{\text {eff }}$ and $\log g$ correctly? 2) Are we interpreting $T_{\text {eff }}$ and $\log g$ correctly?

For the published ELM Survey sample, those objects with $10,000<T_{\text {eff }}<20,000 \mathrm{~K}$ are almost certainly ELM WDs. The spectra of these stars are dominated by hydrogen Balmer lines, lines shaped by Stark broadening and thus sensitive to temperature and gravity at the temperature of B-type stars. The detached eclipsing binary J0651 proves the point: its radial velocity orbital solution, eclipse light curve, and spectroscopic stellar atmosphere parameters all demonstrate that this is a $M=0.25 M_{\odot}, R=0.037 R_{\odot}$ ELM WD orbiting another WD (Brown et al. 2011; Hermes et al. 2012a). The same is also true for two additional eclipsing binary systems, GALEX J1717+6757 (Vennes et al. 2011) and SDSS J0751-0141 (Kilic et al. 2014).

The situation changes between $\mathrm{A} 0$ and F0 spectral types at cooler temperatures $(10,000 \mathrm{~K}$ to $7500 \mathrm{~K})$ where the sdA stars are found. Metal line blanketing is important below 10,000 K, and the Balmer lines become insensitive to temperature (Strom 1969). Most metals have ionization potentials of $5-8 \mathrm{eV}$ that are lower than $\mathrm{H}(13.5 \mathrm{eV})$, and so, despite being rare, metals can contribute a significant fraction of electron pressure in the atmosphere of A-type stars (Strömgren 1969). While we would expect metallicities of A-type main sequence stars to be low - such stars would be at $\sim 10 \mathrm{kpc}$ depths in the halo in our magnitude-limited survey - about one-sixth of A-type stars are chemically peculiar and enhanced in metals (Abt \& Morrell 1995). Helium is a particularly problematic element because its abundance is difficult to measure in A-type spectra. The ionization potential of $\mathrm{He}$ is higher than $\mathrm{H}$, which means He does not add to electron pressure or opacity at A-type temperatures, but it does add to the weight of the atmosphere (Strömgren et al. 1944).

Spectroscopic analysis of the metal-rich WD GD 362 provides a telling example. Based on low-resolution spectra and pure hydrogen atmosphere models, Gianninas et al. (2004) derive an erroneously high $\log g=9.1$ for this star. Follow-up high resolution spectroscopy 
and parallax observations demonstrate that GD 362 has a helium-dominated atmosphere and $\log g=8.2$ (Zuckerman et al. 2007; Kilic et al. 2008).

Yet another complication is that the classic instability strip crosses the main sequence at A- and F-type temperatures. About one-third of A-type stars are observed to be $\delta$ Scuti variables, or SX Phe variables if they are metal-poor (Breger 2000). Fitting models to spectra is also complicated by the fact that the blackbody peak moves across the Balmer discontinuity at A- and F-type temperatures. Thus there are many reasons to be cautious about deriving the parameters of sdA stars.

We constrain the physical nature of sdA stars with a three-pronged approach. First, we compare the observed distributions of WDs and sdA stars. Second, we fit pure hydrogen models to synthetic main sequence spectra to quantify systematic error in the derived temperature and gravity. We also compare alternative SEGUE Stellar Parameter Pipeline fits to sdA spectra. Third, we study in detail a sample of $11 \mathrm{sdA}$ stars suspected of being eclipsing binaries (Kepler 2015, private communication) and 11 previously unpublished ELM WD candidates with sdA-like temperatures. Most of the stars in this sample have significant radial velocity variability; 6 objects have well-measured eclipse light curves; 5 objects have significant infrared excess. A joint constraint on the observations suggests that sdA stars are metal-poor A-F type main sequence stars with late-type companions.

\section{SURVEYS}

We begin by introducing the published samples of sdA stars and ELM WDs. A comparison of basic observational parameters shows that sdA stars differ systematically from ELM WDs in color (temperature) and in reduced proper motion (luminosity).

\section{1. sdA Star Sample}

The sample of sdA stars comes from the SDSS WD catalogs of Kepler et al. (2015, 2016). SDSS acquires spectra using a complicated target selection that depends on magnitude, color, proper motion, and position as summarized by Kepler et al. (2015, 2016). Kepler et al. (2015, 2016) fit pure hydrogen and pure helium atmosphere models to these SDSS spectra to identify WDs. Because of the underlying target selection, the catalog of SDSS WDs is incomplete in magnitude, color, proper motion, and position.

Figure 1 plots the distribution of $T_{\text {eff }}$ and $\log g$ derived from the pure hydrogen and pure helium model atmosphere fits (Kepler et al. 2015, 2016). We draw Figure 1 like an H-R diagram, with temperature increasing to the

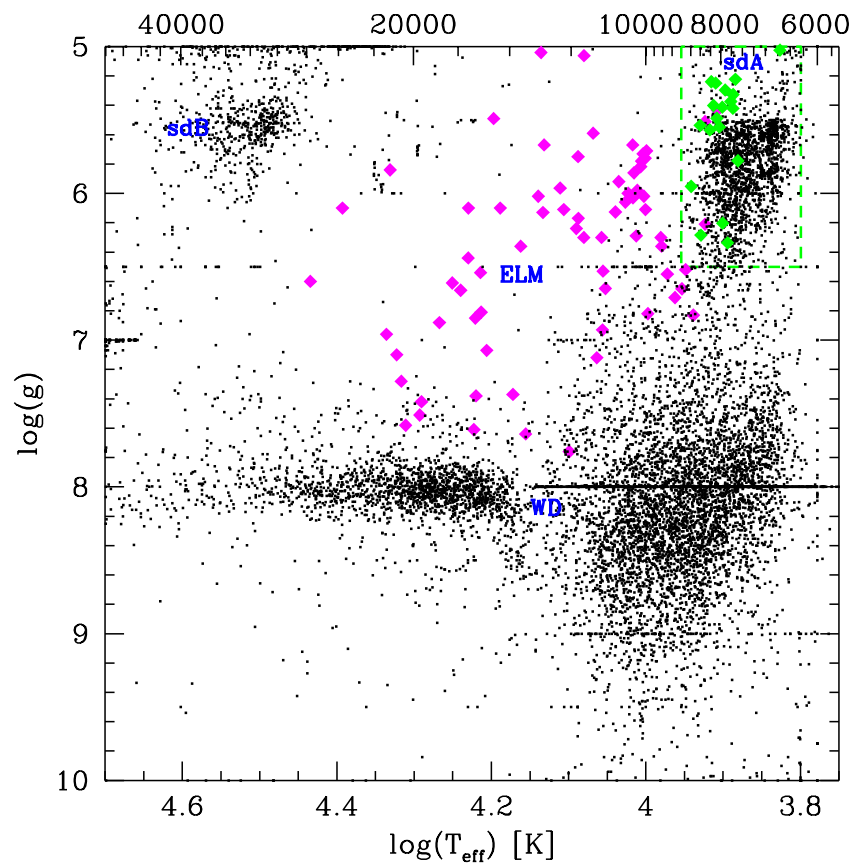

Figure 1. Distribution of Kepler et al. $(2015,2016) T_{\text {eff }}$ and $\log g$ derived from pure hydrogen and pure helium model fits to SDSS spectra (black dots). Clouds of normal sdB stars and WDs are labeled; the cloud of sdA stars is marked by the green dashed rectangle. Published ELM WD binaries are over-plotted as magenta diamonds. Green diamonds mark the sdA stars studied here.

left and gravity decreasing (and thus luminosity increasing) to the top. The largest cloud of objects are normal DA and DB WDs scattered around $\log g=8$. Horizontal lines at fixed $\log g$ are DC and DZ WDs for which only temperature can be measured. There is also a small cloud of objects with $\log g=5.5$ around $30,000 \mathrm{~K}$ which are the helium-burning subdwarf B stars. Low mass WD binaries published in the ELM Survey, described below, are marked by magenta diamonds.

Figure 1 shows a significant, and unexpected, cloud of objects with $\log g \sim 6$ at cool $T_{\text {eff }}<9000 \mathrm{~K}$ temperatures: the sdA stars. Given the observed distribution, we define sdA stars as having $6500 \mathrm{~K}<T_{\text {eff }}<9000 \mathrm{~K}$, $5<\log g<6.5$ (green dashed box) for the purposes of this paper. There are about 2600 sdA stars in this sample. Some of these sdA stars are binaries, systems that allow us to place physical constraints on the nature of sdA stars. The green diamonds mark the subset of sdA stars that we study in greater detail below.

\subsection{ELM WD Sample}

The sample of ELM WDs comes from the ELM Survey (Kilic et al. 2010, 2011, 2012; Brown et al. 2010, 2012, 2013, 2016a; Gianninas et al. 2015). The ELM Survey is a targeted spectroscopic survey of low mass WD can- 


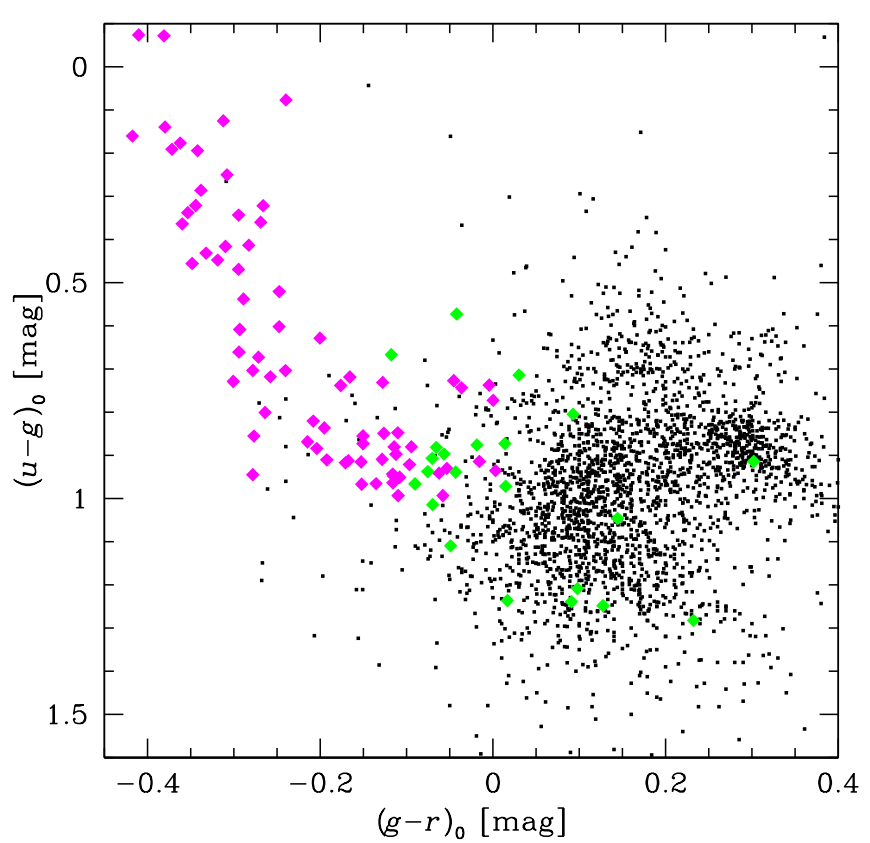

Figure 2. De-reddened $(g-r)$ versus $(u-g)$ color-color plot for the sdA stars (black dots) inside the green dashed box in Fig. 1, and published ELM WDs (magenta diamonds). Green diamonds mark the sdA stars studied here.

didates selected by color. The sample is complete in magnitude $15<g<20$ over the entire SDSS imaging footprint; stars with all proper motions are observed. We focus our attention on the 76 published ELM WDs found in single-lined spectroscopic binaries, objects for which we have physical constraints on the nature of the stars.

Figure 2 plots the distribution of de-reddened $(g-r)_{0}$ and $(u-g)_{0}$ color for the ELM WD binaries (magenta diamonds) and the sdA stars (black dots, those inside the green dashed box from Figure 1). We draw the Figure like an $\mathrm{H}-\mathrm{R}$ diagram again, with temperature increasing to the left. The band of ELM WD binaries reflects the ELM Survey color selection: we target stars with midto late-B type colors, so that is what we find.

The cloud of sdA stars in Figure 2 is systematically redder than the ELM WDs. The difference in $(g-r)_{0}$ color indicates that sdA stars are systematically cooler than published ELM WDs. The green diamonds in Figure 2 again mark the subset of sdA stars that we study in greater detail below.

\subsection{Reduced Proper Motion Comparison}

Reduced proper motion is a classic tool for separating stars at the same temperature by their luminosity (e.g. subdwarfs, dwarfs, and giants). Gentile Fusillo et al. (2015) apply this technique to the SDSS photometric

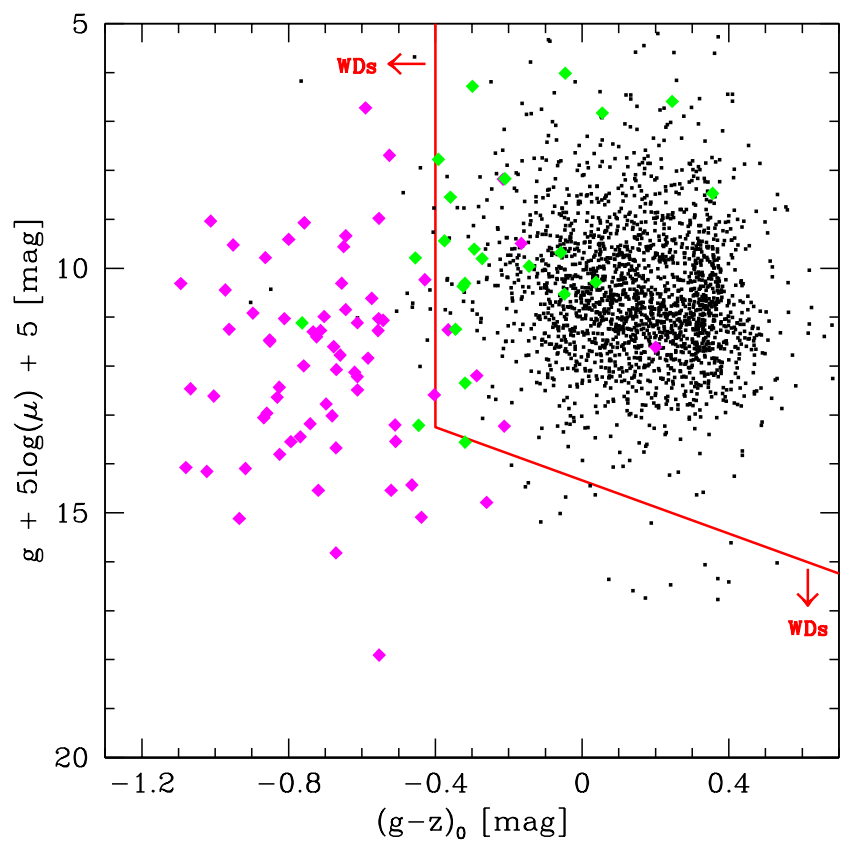

Figure 3. Reduced proper motion versus de-reddened ( $g-$ $z$ ) color for the sdA stars (black dots) inside the green dashed box in Fig. 1, and published ELM WDs (magenta diamonds). Solid red line marks the division between SDSS WDs (lower left) and quasars (upper right) seen by Gentile Fusillo et al. (2015). Green diamonds mark the sdA stars studied here.

catalog and find a clean separation between main sequence stars and WDs using a color-RPM diagram (see also Kilic et al. 2006). We copy their approach for sdA stars and ELM WDs.

We use proper motions, $\mu$, obtained from the HSOY catalog (Altmann et al. 2017), a new proper motion catalog that uses Gaia Data Release 1 positions for its final epoch. Figure 3 plots the distribution of reduced proper motion, $H_{g}=g+5 \log (\mu)+5$, versus de-reddened $(g-z)_{0}$ color. As before, sdA stars are plotted as black dots, and ELM WDs are plotted as magenta diamonds. The solid red line marks the division between quasars (luminous distant objects), which sit in the upper right, and WDs (faint nearby objects), which sit below and to the left (Gentile Fusillo et al. 2015).

Gentile Fusillo et al. (2015) observe WDs with $(g-$ $z)_{0} \simeq 0$ colors similar to the sdA stars, but the WDs are intrinsically fainter, $H_{g}>15 \mathrm{mag}$, and fall below the red line. Thus Figure 3 shows that sdA stars are systematically more luminous for their color (temperature) than WDs; $98 \%$ of sdA stars lie above the red line in Figure 3. Conversely, the ELM WDs have luminosities of known WDs at comparable temperatures; $92 \%$ of published ELM WDs lie below or to the left of the red line in Figure 3. 


\section{STELLAR PARAMETERS}

In the previous section we find that sdA stars have cooler temperatures and greater luminosities than published ELM WDs. The luminosity of a star is related to its radius and effective temperature according to the Stephan-Boltzmann law,

$$
L=4 \pi R^{2} \sigma T^{4}
$$

where $L$ is luminosity, $R$ is radius, $\sigma$ is the StephanBoltzmann constant, and $T$ is effective temperature. Equation 1 tells us that, for sdA stars to be cooler and more luminous than ELM WDs, they must have larger radii.

Surface gravity depends on mass $M$ divided by radius squared,

$$
\log g=4.438+\log \left(M / R^{2}\right),
$$

where $g$ is in units of $\mathrm{cm} \mathrm{s}^{-2}, M$ is in units of solar mass, and $R$ is units of solar radii. Equation 2 tells us that a typical $\log g=6, M=0.2 M_{\odot}$ ELM WD should have a radius of $0.074 R_{\odot}$. Such radii are observed for ELM WDs (Hermes et al. 2014).

An sdA star that has the same surface gravity but a larger radius than an ELM WD must have a larger mass. Quantitatively, a 3 times larger radius requires a 9 times larger mass, or $1.8 M_{\odot}$ to keep surface gravity constant. $1.8 M_{\odot}$ is the mass of an A-type main-sequence star, but $\log g=6$ is not the correct gravity for a main-sequence star.

One explanation is that sdA surface gravities derived from pure hydrogen models are incorrect. Metal line blanketing, combined with the insensitivity of the hydrogen Balmer lines at these temperatures, may cause the pure hydrogen models to yield incorrect parameters. We test this hypothesis in two ways.

\subsection{Theoretical Test}

First, we fit our pure hydrogen model atmospheres to synthetic spectra of main sequence B-, A-, and F0-type stars. We generate synthetic spectra using ATLAS9 ODFNEW model atmosphere grids (Castelli \& Kurucz 2004; Castelli et al. 1997) convolved to the $1 \AA$ spectral resolution of our MMT Blue Channel Spectrograph data. We fix $\log g=4.5$ and step $T_{\text {eff }}$ from $7500 \mathrm{~K}$ to $15,000 \mathrm{~K}$ at solar metallicity and at one-tenth solar metallicity $([\mathrm{M} / \mathrm{H}]=-1)$. We also set $v \sin i=100$ $\mathrm{km} \mathrm{s}^{-1}$ appropriate for main sequence stars of these temperatures (Abt et al. 2002). Setting $v \sin i=0 \mathrm{~km} \mathrm{~s}^{-1}$ changes the results very little, by less than $1 \%$.

We then fit the synthetic spectra to the Gianninas et al. (2011, 2014a) grid of pure hydrogen atmosphere models used for the ELM Survey. Tremblay et al. (2013, 2015)

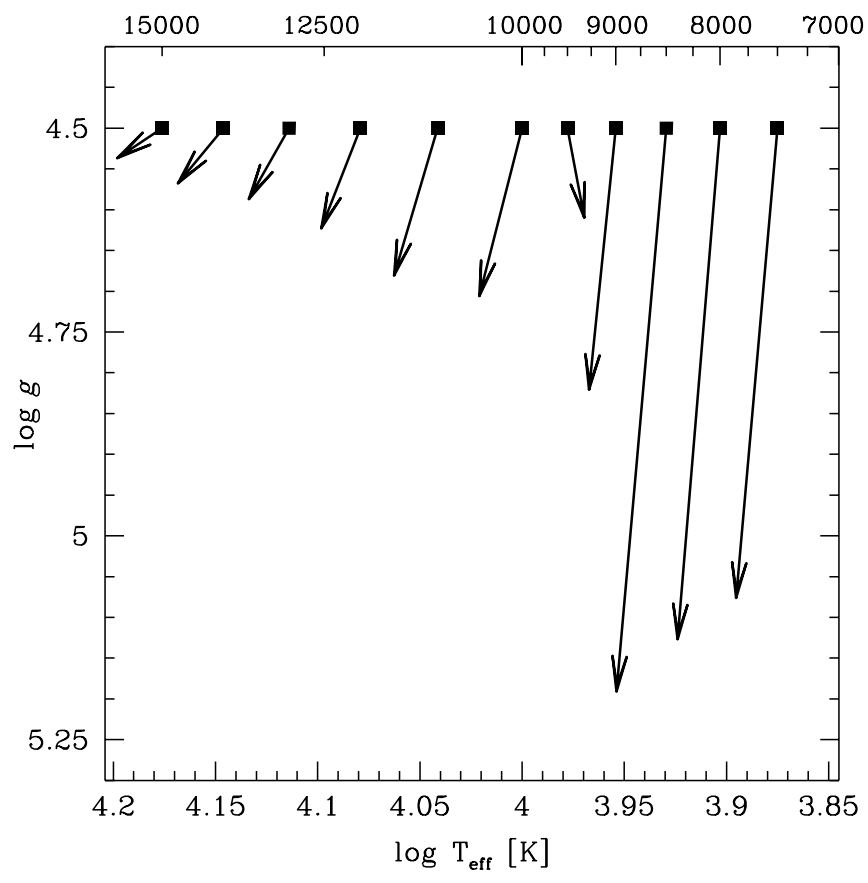

Figure 4. Systematic error of pure hydrogen model fits (arrows) to synthetic main sequence spectra with known parameters (black squares). For $[\mathrm{M} / \mathrm{H}]=-1$, shown here, the systematic error in $\log g$ is about 0.6 dex below $9000 \mathrm{~K}$.

show that parameters obtained from 1D stellar atmosphere fits must be corrected for 3D convection effects, and provide $1 \mathrm{D}$ to $3 \mathrm{D}$ corrections for the range $6000 \mathrm{~K}$ $<T_{\text {eff }}<14,000 \mathrm{~K}$ and $5<\log g<9$. Only our coolest $<9000 \mathrm{~K}$ synthetic spectra have fitted parameters that rise above $\log g=5$ and fall in this range. The predicted corrections would reduce the fitted $T_{\text {eff's }}$ by about $5 \%$ and $\log g$ 's by about 0.1 dex. However, because we do not have corrections for most of the measurements, we do not apply any corrections. Our immediate interest is making a self-consistent comparison.

Figure 4 presents the results for the $[\mathrm{M} / \mathrm{H}]=-1$ synthetic spectra. We find that $T_{\text {eff }}$ fit from the pure hydrogen atmosphere models is systematically too large by about $5 \%$ across all temperatures. It is possible that an appropriate $1 \mathrm{D}$ to $3 \mathrm{D}$ correction could address this. Surface gravity shows a similar systematic error above $9000 \mathrm{~K}$, however the error in $\log g$ explodes to 0.6 dex at sdA temperatures below $9000 \mathrm{~K}$. This is many times larger than tabulated $1 \mathrm{D}$ to $3 \mathrm{D}$ corrections (Tremblay et al. 2015). If we look at the results for the solar metallicity synthetic spectra, we find that the systematic error in $\log g$ is even greater below $9000 \mathrm{~K}$, nearly 1.0 dex. We conclude that pure hydrogen models systematically over-estimate the $\log g$ of main sequence spectra at sdA temperatures below about $9000 \mathrm{~K}$. 


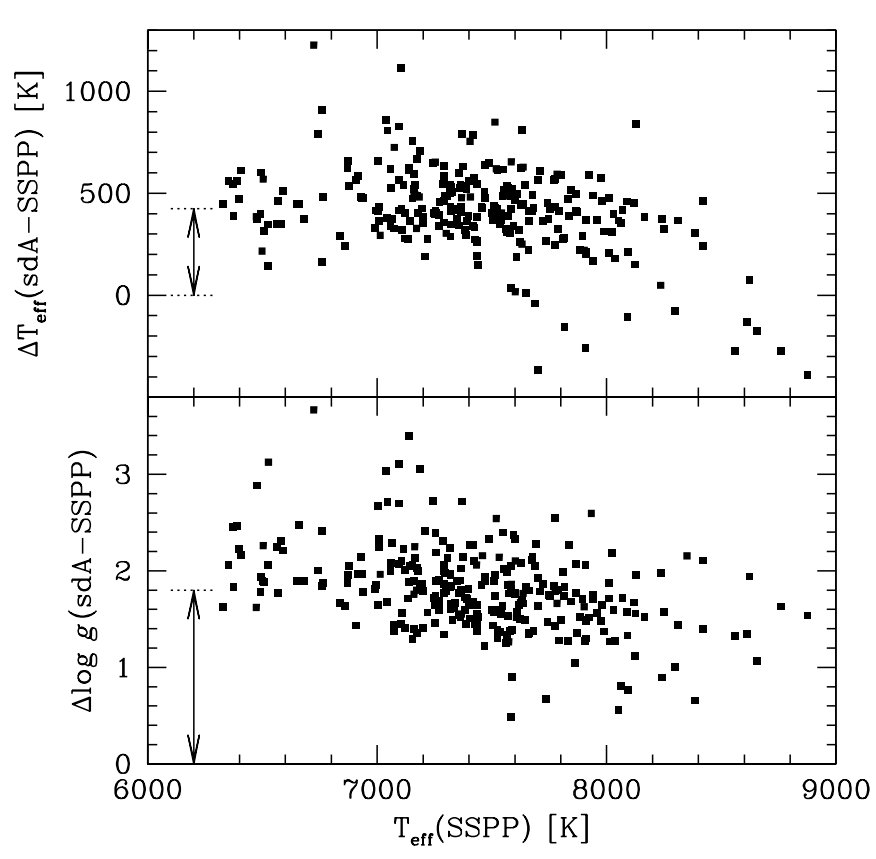

Figure 5. Difference between Kepler et al. (2015, 2016) pure hydrogen model fits and SSPP main sequence fits to sdA stars (black dots) inside the green dashed box in Fig. 1, plotted as a function of SSPP effective temperature.

\subsection{Empirical Test}

Second, we compare two different fits to the same observed spectra. We cross-match the list of sdA stars (Kepler et al. 2015, 2016) against the SEGUE Stellar Parameter Pipeline (SSPP, Lee et al. 2008; Allende Prieto et al. 2008; Smolinski et al. 2011) and find 287 matches. This is about $10 \%$ of the sdA list; the other sdA stars are from SDSS spectra obtained after Data Release 9 and so are not included in the SSPP catalog.

Figure 5 plots the difference between the pure hydrogen atmosphere model fits (Kepler et al. 2015, 2016) and the SSPP main sequence fits as a function of SSPP temperature. There is a systematic offset: the median pure hydrogen atmosphere $T_{\text {eff }}$ is $425 \mathrm{~K}$ hotter (or $5 \%$ at $8000 \mathrm{~K}$, the same as the synthetic tests), and the median $\log g$ is 1.8 dex larger than the SSPP main sequence model.

The sdA stars have a median $\log g \simeq 4$ in the SSPP catalog; this is the surface gravity of a main sequence star. The sdA stars have a median $[\mathrm{Fe} / \mathrm{H}]=-1.5$ in the SSPP catalog; this is the metallicity of a halo star (Allende Prieto et al. 2014).

Metal-poor stars are less luminous and lower mass than solar-metallicity stars of identical temperature. Consider an $8000 \mathrm{~K}$ star. In the Padova main sequence tracks (Bressan et al. 2012) an $8000 \mathrm{~K}$ star has $1.9 M_{\odot}$ and $2.5 R_{\odot}$ at solar metallicity, or $1.2 M_{\odot}$ and $1.1 R_{\odot}$ at $[\mathrm{M} / \mathrm{H}]=-1$. Thus a metal-poor $8000 \mathrm{~K}$ star is near the main sequence turn-off in the halo; hotter stars may be halo blue stragglers. For comparison, an $8000 \mathrm{~K}$ ELM WD has $0.18 M_{\odot}$ and $0.03 R_{\odot}$ (Althaus et al. 2013; Istrate et al. 2016).

\section{NEW OBSERVATIONS}

To test whether or not sdA stars are indeed metalpoor main sequence stars, we study a sub-sample of sdA stars in greater detail. Our sample is comprised of 11 sdA stars suspected of being eclipsing binaries (Kepler 2015 , private communication) and 11 previously unpublished ELM WD candidates that have sdA-like temperatures summarized in Table 1. We obtain time-series spectroscopy for all 22 objects and time-series optical photometry for 21 objects. We also obtain JHK infrared photometry for 6 objects. We find that 17 objects are radial velocity variable, 6 are eclipsing, and 4 of these have significant infrared excess. All of these objects are identified with their full coordinates in Table 1.

\subsection{Spectroscopy}

We obtain time-series spectroscopy for 20 of the 22 objects with the $6.5 \mathrm{~m}$ MMT telescope. We configured the Blue Channel spectrograph (Schmidt et al. 1989) to obtain 1.0 A resolution spectra, and set exposure times to yield signal-to-noise ratios of 10 to 15 in the continuum. We obtain spectra for the 2 brightest objects with the $1.5 \mathrm{~m}$ Tillinghast telescope at Fred Lawrence Whipple Observatory. We configured the FAST spectrograph (Fabricant et al. 1998) to obtain $1.7 \AA$ resolution spectra, and set exposure times to yield similar signal-to-noise ratios. We obtain additional spectra for 6 objects with the $4 \mathrm{~m}$ Mayall telescope at Kitt Peak National Observatory. We configured the KOSMOS spectrograph (Martini et al. 2014) to obtain 2.0 Å resolution spectra. The spectra were mostly acquired in observing runs between December 2014 and December 2016.

All spectra are paired with comparison lamp exposures for accurate wavelength calibration. We measure radial velocities using the RVSAO cross-correlation program (Kurtz \& Mink 1998). For each object we typically obtain multiple observations on back-to-back nights in different observing runs. This observing pattern allows use to constrain the orbital properties of binaries that have periods ranging from hours to days.

\subsection{Stellar Atmosphere Fits}

We begin by summing the rest-frame spectra and performing stellar atmosphere fits to each object. Our approach is identical to previous ELM Survey papers. In 
Table 1. Summary of Observations

\begin{tabular}{|c|c|c|c|c|c|}
\hline Object & $N_{\text {spec }}$ & RV Variable & $N_{\text {phot }}$ & Eclipsing & IR Excess \\
\hline \multicolumn{6}{|c|}{ sdA stars } \\
\hline SDSS J074735.035+455420.02 & 6 & $\cdots$ & 351 & $\cdots$ & $\cdots$ \\
\hline SDSS J075017.359+400441.30 & 14 & YES & 432 & YES & $\cdots$ \\
\hline SDSS J075804.596+475406.10 & 16 & $\cdots$ & 293 & $\cdots$ & $\cdots$ \\
\hline SDSS J080205.902+433228.29 & 10 & $\cdots$ & 407 & YES & YES \\
\hline SDSS J080313.312+415740.49 & 12 & YES & 421 & YES & $\cdots$ \\
\hline SDSS J080313.957+383037.73 & 10 & YES & 457 & $\cdots$ & $\cdots$ \\
\hline SDSS J080540.813+424350.70 & 8 & YES & 393 & $\cdots$ & $\cdots$ \\
\hline SDSS J082328.325+373101.69 & 8 & YES & 757 & YES & $\cdots$ \\
\hline SDSS J083238.894+135120.98 & 17 & $\cdots$ & 527 & YES & YES \\
\hline SDSS J094154.305+370140.92 & 10 & YES & 373 & $\cdots$ & $\cdots$ \\
\hline SDSS J101132.732+024216.47 & 5 & $\cdots$ & 337 & $\cdots$ & YES \\
\hline \multicolumn{6}{|c|}{ sdA-like ELM WD candidates } \\
\hline SDSS J024318.073-084749.67 & 8 & YES & 243 & $\cdots$ & $\cdots$ \\
\hline SDSS J042128.509+000213.44 & 27 & YES & 339 & $\cdots$ & YES \\
\hline SDSS J081115.372-021652.75 & 16 & YES & 147 & $\cdots$ & $\cdots$ \\
\hline SDSS J091157.257+583308.39 & 6 & YES & 145 & $\cdots$ & $\cdots$ \\
\hline SDSS J140608.922+044302.12 & 11 & YES & 348 & $\cdots$ & YES \\
\hline SDSS J154102.488+314003.68 & 13 & YES & 320 & $\cdots$ & $\cdots$ \\
\hline SDSS J160036.831+272117.81 & 20 & YES & 397 & YES & YES \\
\hline SDSS J160050.256+051617.27 & 19 & YES & 345 & $\cdots$ & $\cdots$ \\
\hline SDSS J205219.372-033208.71 & 5 & YES & 0 & $\cdots$ & $\cdots$ \\
\hline SDSS J220133.319+242005.63 & 4 & $\cdots$ & 118 & $\cdots$ & $\cdots$ \\
\hline SDSS J221928.482+120418.68 & 27 & YES & 473 & $\ldots$ & $\ldots$ \\
\hline
\end{tabular}

brief, we fit the summed spectra to a grid of pure hydrogen atmosphere models that span $4000 \mathrm{~K}<T_{\text {eff }}<$ $35,000 \mathrm{~K}$ and $4.5<\log g<9.5$ (Gianninas et al. 2011, 2014b, 2015). We apply the Tremblay et al. (2013, 2015) $1 \mathrm{D}$ to $3 \mathrm{D}$ corrections to all of the temperatures and gravities (however we are forced to extrapolate the correction for the three objects with $\log g<5)$. We present the final parameters in Table 2, along with the parameters independently published by Kepler et al. (2015, 2016) and by the SSPP catalog, when available.

We find good agreement between the values derived from the two pure hydrogen models, labeled "Gianninas" and "Kepler" in Table 2. Clipping one outlier (J0941+37), the temperatures differ on average by $6 \mathrm{~K}$ with a root-mean-square (RMS) dispersion of $\pm 216 \mathrm{~K}$. The gravities differ on average by 0.20 dex with an RMS dispersion of \pm 0.23 dex. The dispersions are consistent with the errors summed in quadrature. Thus we conclude that the two sets of hydrogen models give consistent answers.
The SSPP parameters are systematically different, however, about the same as before (Section 3.2). The SSPP temperatures for these objects are on average $430 \mathrm{~K}$ cooler than our hydrogen model values, and the gravities are 1.7 dex lower. Surface gravity remains the most divergent parameter, and thus the most suspect. The offsets are in the same direction, and of similar magnitude, as we found from fitting hydrogen models to synthetic main sequence spectra.

\subsection{Radial Velocities}

Our time-series spectroscopy provides a constraint on the radial velocity variability of the 22 objects. We start with an F-test, to test whether the variance of the observed radial velocities exceeds the variance expected from measurement errors for an object at rest. Seventeen objects exhibit significant radial velocity variability at better than $99 \%$ confidence, and thus are very likely binaries. The large number of binaries is expected: half of the objects are candidate eclipsing binaries. We iden- 
Table 2. Stellar Atmosphere Parameters

\begin{tabular}{|c|c|c|c|c|c|c|c|}
\hline \multirow[b]{2}{*}{ Object } & \multicolumn{2}{|c|}{ Gianninas } & \multicolumn{2}{|c|}{ Kepler } & \multicolumn{3}{|c|}{ SEGUE Stellar Parameter Pipeline } \\
\hline & $\begin{array}{l}T_{\text {eff }} \\
(K)\end{array}$ & $\log g$ & $\begin{array}{l}T_{\text {eff }} \\
(\mathrm{K})\end{array}$ & $\log g$ & $\begin{array}{l}T_{\text {eff }} \\
(K)\end{array}$ & $\log g$ & {$[\mathrm{Fe} / \mathrm{H}]$} \\
\hline \multicolumn{8}{|c|}{ sdA stars } \\
\hline $\mathrm{J} 0747+45$ & $7524 \pm 113$ & $5.526 \pm 0.082$ & 7546 & 5.749 & $\cdots$ & $\cdots$ & $\cdots$ \\
\hline $\mathrm{J} 0750+40$ & $7998 \pm 124$ & $5.335 \pm 0.091$ & 8141 & 5.619 & $7524 \pm 46$ & $4.229 \pm 0.155$ & $-0.507 \pm 0.100$ \\
\hline J0758+47 & $7827 \pm 134$ & $5.298 \pm 0.154$ & $\cdots$ & $\cdots$ & $7360 \pm 65$ & $3.549 \pm 0.359$ & $-0.350 \pm 0.062$ \\
\hline $\mathrm{J} 0802+43$ & $7461 \pm 121$ & $5.004 \pm 0.144$ & $\cdots$ & $\cdots$ & $6769 \pm 64$ & $4.380 \pm 0.098$ & $-0.971 \pm 0.014$ \\
\hline $\mathrm{J} 0803+41$ & $8150 \pm 124$ & $5.345 \pm 0.075$ & 8355 & 5.885 & $\cdots$ & $\cdots$ & $\cdots$ \\
\hline J0803+38 & $7777 \pm 118$ & $6.056 \pm 0.076$ & 7646 & 6.303 & $7686 \pm 204$ & $4.255 \pm 0.171$ & $-0.801 \pm 0.240$ \\
\hline $\mathrm{J} 0805+42$ & $7870 \pm 120$ & $5.909 \pm 0.079$ & 7660 & 6.041 & $7815 \pm 176$ & $4.211 \pm 0.145$ & $-1.086 \pm 0.090$ \\
\hline J0823+37 & $7558 \pm 125$ & $5.180 \pm 0.142$ & 7799 & 5.323 & $7224 \pm 55$ & $4.218 \pm 0.124$ & $-1.010 \pm 0.129$ \\
\hline $\mathrm{J} 0832+13$ & $6652 \pm 111$ & $4.905 \pm 0.178$ & 6786 & 5.359 & $\ldots$ & $\ldots$ & $\ldots$ \\
\hline $\mathrm{J} 0941+37$ & $8337 \pm 130$ & $5.982 \pm 0.084$ & 7646 & 5.880 & $7906 \pm 183$ & $3.825 \pm 0.184$ & $-1.697 \pm 0.095$ \\
\hline $\mathrm{J} 1011+02$ & $8466 \pm 147$ & $5.722 \pm 0.128$ & 8109 & 5.549 & $7884 \pm 51$ & $4.030 \pm 0.210$ & $-1.220 \pm 0.102$ \\
\hline
\end{tabular}

sdA-like ELM WD candidates

\begin{tabular}{cccccccc}
\hline $\mathrm{J} 0243-08$ & $8172 \pm 138$ & $5.402 \pm 0.131$ & $\ldots$ & $\ldots$ & $\ldots$ & $\ldots$ & $\ldots$ \\
$\mathrm{J} 0421+00$ & $8093 \pm 120$ & $5.488 \pm 0.063$ & $\ldots$ & $\ldots$ & $\ldots$ & $\ldots$ & $\ldots$ \\
$\mathrm{J} 0811-02$ & $7709 \pm 116$ & $5.326 \pm 0.083$ & $\ldots$ & $\ldots$ & $\ldots$ & $\ldots$ & $\ldots$ \\
$\mathrm{J} 0911+58$ & $7961 \pm 154$ & $5.413 \pm 0.192$ & $\ldots$ & $\ldots$ & $\ldots$ & $\ldots$ & $\ldots$ \\
$\mathrm{J} 1406+04$ & $8119 \pm 131$ & $5.249 \pm 0.110$ & $\ldots$ & $\ldots$ & $\ldots$ & $\ldots$ & $\ldots$ \\
$\mathrm{J} 1541+31$ & $7891 \pm 119$ & $5.296 \pm 0.078$ & $\ldots$ & $\ldots$ & $\ldots$ & $\ldots$ & $\ldots$ \\
$\mathrm{J} 1600+27$ & $7910 \pm 138$ & $4.775 \pm 0.203$ & $\ldots$ & $\ldots$ & $\ldots$ & $\ldots$ & $\ldots$ \\
$\mathrm{J} 1600+05$ & $7762 \pm 115$ & $5.374 \pm 0.069$ & $\ldots$ & $\ldots$ & $7794 \pm 286$ & $4.261 \pm 0.242$ & $-1.797 \pm 0.114$ \\
$\mathrm{~J} 2052-03$ & $8081 \pm 140$ & $4.864 \pm 0.169$ & $\ldots$ & $\ldots$ & $\ldots$ & $\ldots$ & $\ldots$ \\
$\mathrm{J} 2201+24$ & $8397 \pm 232$ & $4.751 \pm 0.511$ & $\ldots$ & $\ldots$ & $\ldots$ & $\ldots$ & $\ldots$ \\
$\mathrm{J} 2219+12$ & $8226 \pm 127$ & $5.240 \pm 0.073$ & $\ldots$ & $\ldots$ & $\ldots$ & $\ldots$ & $\ldots$ \\
\hline
\end{tabular}

Note-Gianninas values are corrected for 3D convection effects following Tremblay et al. (2013, 2015). Kepler values come from Kepler et al. (2015, 2016).

tify the velocity variable objects in Table 1, and provide the individual measurements in the Appendix.

We calculate orbital elements for the velocity variable objects using the same procedure as in previous ELM Survey papers. In brief, we minimize $\chi^{2}$ for circular orbits using the code of Kenyon \& Garcia (1986). We search for orbital periods up to 4 days, which is the maximum time span of our individual observing runs.

Six objects have well-constrained orbital parameters; the remaining velocity variable objects have insufficient observations or phase coverage to yield unique orbital solutions. We plot the periodograms and the phased radial velocities for the well-constrained objects in Figure 6.

We estimate the significance of possible orbital period aliases for these six objects using the $\chi^{2}$ values.
For normally distributed errors, a $\Delta \chi^{2}=13.3$ with respect to the minimum value corresponds to a $99 \%$ confidence interval for 4 degrees of freedom (Press et al. 1992). On this basis, three objects have no significant period aliases: J0421+00 is $P=16.94 \mathrm{hr}$, J0750+40 is $P=28.35 \mathrm{hr}$, and $\mathrm{J} 0803+41$ is $P=32.27 \mathrm{hr}$. Three other objects have significant but uninteresting period aliases: J0811-02 is $P=33.8 \mathrm{hr}$ with a cloud of aliases between $31.7 \mathrm{hr}$ and $35.3 \mathrm{hr}$, J1541+31 is either $P=16.8 \mathrm{hr}$ or $29 \mathrm{hr}$, and $\mathrm{J} 1600+27$ is either $P=23.10$ or $24.08 \mathrm{hr}$. We present the orbital parameters for these six objects in Table 3 .

Unlike published ELM WD binaries, we find no evidence for short-period or high-amplitude velocity variability in any of the 22 sdA-like objects studied here. 

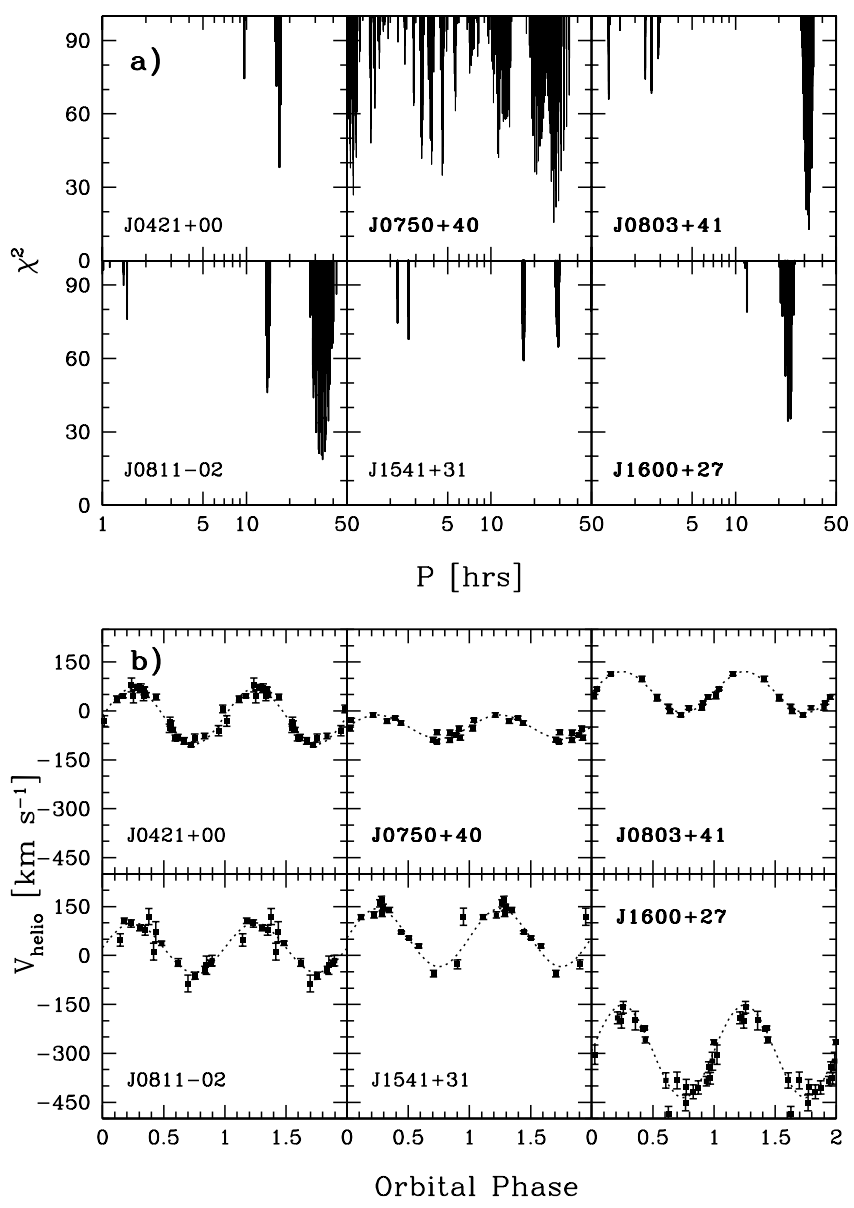

Figure 6. (a) Periodograms for 6 objects with wellconstrained orbital solutions (upper panel). (b) Heliocentric radial velocities phased to the best-fit periods.

The day-long orbital periods that we do observe are confirmed by light curves.

\subsection{Light Curves}

We search the Catalina Surveys Data Release 2 (Drake et al. 2009) and find time-series $V$-band photometry for 21 of the 22 objects. Six objects show significant eclipses. Three of the eclipsing systems have orbital periods in excellent agreement with their radial velocity solutions. We adopt the photometric periods for these systems (J0750+40, J0803+41, and J1600+27).

Figure 7 displays the Catalina light curves and our best-fit model for the six eclipsing systems. We model the light curves using JKTEBOP (Southworth et al. 2004). Based on our initial fits, all six binary systems are composed of stars with radii consistent within a factor of two. Hence, we assume a mass ratio of 1 in our light curve fits and use the appropriate gravity and linear limb darkening coefficients for $T_{\text {eff }}=8000 \mathrm{~K}, \log g=4$, and solar metallicity stars from Claret \& Bloemen (2011).
Table 3. Radial Velocity Orbital Fits

\begin{tabular}{ccccr}
\hline \hline Object & $P$ & alias & $k$ & \multicolumn{1}{c}{$\gamma$} \\
& $(\mathrm{d})$ & $(\mathrm{d})$ & $\left(\mathrm{km} \mathrm{s}^{-1}\right)$ & \multicolumn{1}{c}{$\left(\mathrm{km} \mathrm{s}^{-1}\right)$} \\
\hline J0421+00 & 0.705822 & none & $86.2_{-3.3}^{+3.2}$ & $-15.8 \pm 2.7$ \\
J0750+40 & 1.181426 & none & $36.2_{-10.6}^{+10.5}$ & $-48.8 \pm 8.9$ \\
J0803+41 & 1.344415 & none & $63.1_{-17.5}^{+12.0}$ & $57.2 \pm 8.9$ \\
J0811-02 & 1.407864 & 1.32 to 1.47 & $76.5_{-10.1}^{+7.8}$ & $23.9 \pm 11.2$ \\
J1541+31 & 0.698590 & 1.217964 & $88.8_{-7.8}^{+5.4}$ & $54.9 \pm 24.5$ \\
J1600+27 & 1.003368 & 0.962638 & $142.0_{-52.2}^{+33.9}$ & $-293.7 \pm 52.0$ \\
\hline
\end{tabular}

We perform 100 Monte Carlo simulations for each star to estimate the errors in each parameter.

Table 4 presents our best-fit model parameters for the six eclipsing systems, whose periods range from 0.7 to 3.7 days. It is striking that all six systems include binary stars with radii that range from $10 \%$ to $\approx 30 \%$ of the orbital separation. These stars are too large to be WDs; they are clearly main-sequence stars. Note that the average radius of the primary star in J0802+4332 is nearly $30 \%$ of the orbital separation, so this radius may be wrong by $5 \%$ (North \& Zahn 2004).

The assumed mass ratio, $q=M_{2} / M_{1}$, has negligible effect on the radii measurements for all but two of the stars, J0802+4332 and J0803+4157. Yet even for $q=0.25$, the radii estimates are larger than $10 \%$ of the orbital separation for all primary and secondary stars in all six systems. Thus our choice of $q$ does not affect the conclusion that these stars are too large to be WDs.

Table 4 also presents the $V$-band luminosity ratios, $L_{2} / L_{1}$, for the eclipsing systems. The secondary eclipses for these six objects are less well defined, compared to the primary eclipses. This results in large uncertainties in the luminosity ratios of the two stars in each system. With the exception of $\mathrm{J} 1600+27$, the eclipse ratios are consistent with luminosity ratios of about ten of percent in the $V$-band.

\subsection{Spectral Energy Distributions}

We search the Two Micron All Sky Survey (2MASS, Skrutskie et al. 2006) and the UKIRT Infrared Deep Sky Survey (UKIDSS, Lawrence et al. 2007) and find nearinfrared photometry in more than one passband for six objects. Figure 8 shows the spectral energy distributions (SEDs) of these six objects. Points with error bars show the observed photometry, whereas the dotted and dashed lines show fiducial spectral templates for A0 to M0 type stars (Pickles 1998).

Five of these stars, excluding J1011+0242, are binary systems with radial velocity variations or photometric 
Table 4. Light Curve Fits for Six Eclipsing Systems

\begin{tabular}{cccccc}
\hline \hline \multirow{2}{*}{ Object } & $i$ & $P$ & $r_{1} / a$ & $r_{2} / a$ & $L_{2} / L_{1}$ \\
& $\left({ }^{\circ}\right)$ & $(\mathrm{d})$ & & & \\
\hline $\mathrm{J} 0750+40$ & $86.8_{-3.4}^{+1.2}$ & $1.181426(2)$ & $0.217_{-0.049}^{+0.014}$ & $0.189_{-0.009}^{+0.028}$ & $0.096_{-0.042}^{+0.141}$ \\
$\mathrm{~J} 0802+43$ & $87.2_{-4.7}^{+1.7}$ & $2.541831(4)$ & $0.297_{-0.038}^{+0.008}$ & $0.231_{-0.006}^{+0.028}$ & $\ldots$ \\
$\mathrm{J} 0803+41$ & $83.0_{-4.3}^{+2.5}$ & $1.344415(4)$ & $0.269_{-0.029}^{+0.025}$ & $0.192_{-0.016}^{+0.050}$ & $\ldots$ \\
$\mathrm{J} 0823+37$ & $86.7_{-4.6}^{+1.7}$ & $0.7339255(7)$ & $0.275_{-0.046}^{+0.012}$ & $0.220_{-0.009}^{+0.031}$ & $0.091_{-0.045}^{+0.125}$ \\
$\mathrm{~J} 0832+13$ & $85.4_{-3.3}^{+2.9}$ & $3.66975(3)$ & $0.226_{-0.017}^{+0.025}$ & $0.107_{-0.010}^{+0.018}$ & $\ldots$ \\
$\mathrm{J} 1600+27$ & $86.2_{-1.7}^{+2.8}$ & $1.003368(2)$ & $0.108_{-0.012}^{+0.007}$ & $0.220_{-0.016}^{+0.013}$ & $1.185_{-0.089}^{+0.101}$ \\
\hline
\end{tabular}

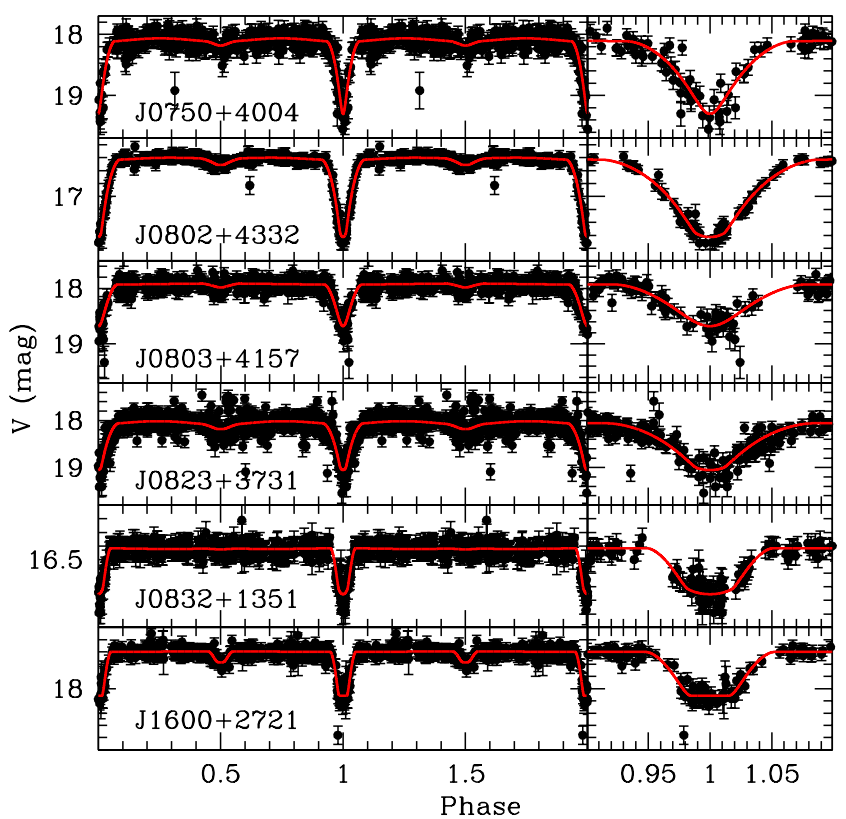

Figure 7. Phased-folded Catalina $V$-band photometry for 6 eclipsing binaries (black points) overplotted with the bestfit light curve models (red lines).

eclipses. Thus we model these SEDs with composite A0 to M0 main-sequence star spectral templates, scaling each spectral template based on its absolute $V$-band magnitude from Table 2 of Pickles (1998). The solid lines show the best-fitting composite template for each system. The SED for J0802+4332 is the most unusual in this sample, as it is brightest in the $J$-band and has the largest residuals. J0802+4332 may require a larger and more evolved companion to explain its infrared photometry.

We note that the spectral types shown in Figure 8 are meant to be representative. The implied mass ratios, based purely on broadband photometry, range from

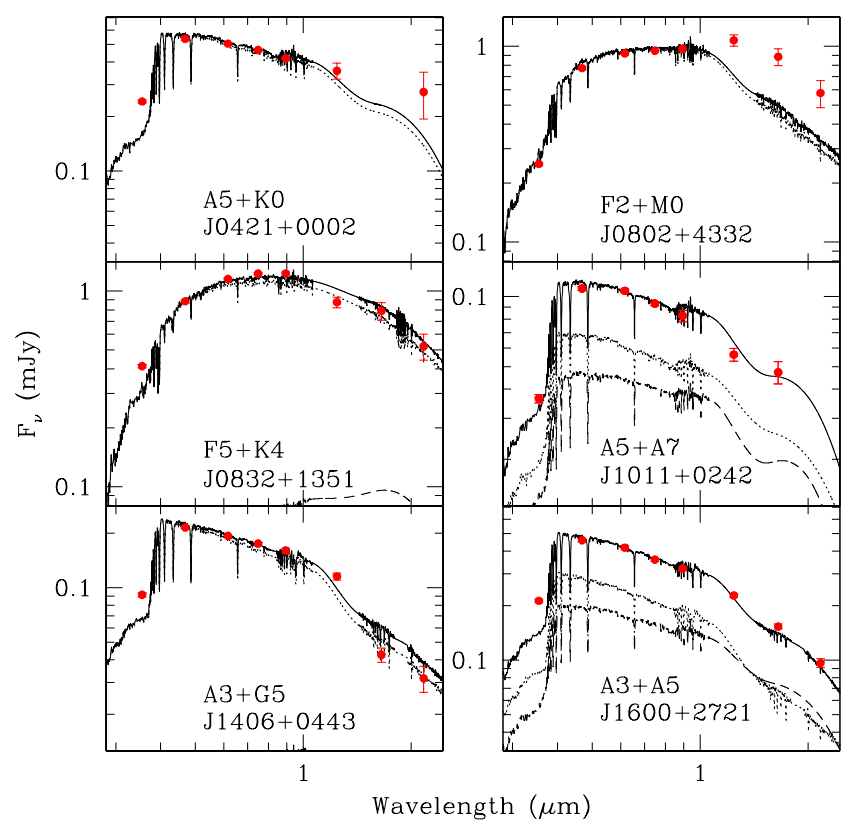

Figure 8. Spectral energy distributions for 6 objects with infrared photometry in more than one passband. The SDSS plus 2MASS or UKIDSS photometry (red points) are compared against fiducial spectral templates (black lines). The spectral types listed here are representative; the next Figure incorporates radial velocity and eclipse information.

$q=0.3$ to 0.9 . Adding the radial velocity and eclipse information allows for more accurate constraints.

\section{RESULTS}

We now combine the data into a joint constraint on the physical nature of sdA stars. Eclipses constrain the ratio of stellar radii and, given the Stephan-Boltzmann Law, the ratio of stellar temperatures. Radial velocities, given Kepler's 3rd Law, constrain the ratio of masses in each binary. Adopting an absolute measurement, such 
as the mass of the primary star, sets the absolute scale for the entire system.

Stellar evolution models provide us with libraries of physically possible tracks of mass, radius, luminosity, and temperature as a function of a star's age. We consider two families of stellar evolution models relevant to these $\log g=4$ to 6 objects: Padova main sequence tracks (Bressan et al. 2012) and helium-core WD tracks (Althaus et al. 2013).

Our goal is to identify the combination of primary and secondary stellar mass, radius, luminosity, and temperature that, together, best match the observations. The observations are orbital period, velocity amplitude, inclination, stellar radius-to-semi-major axis ratio, luminosity ratio, and primary temperature. We ignore our $\log g$ measurements, because surface gravity is the parameter we are trying to understand.

Our approach is to adopt a trial primary mass $M_{1}$, and then calculate the resulting $M_{2}$, stellar radii, surface gravities, and luminosities required by the observations. Stepping $M_{1}$ from $0.1 M_{\odot}$ to $2.5 M_{\odot}$ presents us with the plausible range of solutions for the $T_{\text {eff }} \simeq$ $8000 \mathrm{~K}$ primary stars. The correct solution is the one at which the primary and secondary stellar parameters self-consistently match evolutionary tracks. We consider the possibility that the binaries contain WD+WD, $\mathrm{WD}+\mathrm{MS}, \mathrm{MS}+\mathrm{WD}$, or MS+MS stars.

Some observational quantities are better constrained than others, and so we perform a Monte Carlo calculation to account for the errors. The result of the Monte Carlo calculation is a cloud of possible primary and secondary stellar parameters for each system. For every set of parameters, we record the best-matching pair of points in the respective stellar evolutionary tracks.

\subsection{Joint Constraints}

Figure 9 visualizes the results for the three bestconstrained cases: J0750+40, J0803+41, and J1600+27. All three are $8000 \mathrm{~K}$ objects in eclipsing binaries with radial velocity orbital solutions. We present the results with a pair of plots for each object: temperatureluminosity (left-hand panel) and mass-radius (righthand panel). All plots are drawn on a log-log scale. Thus stellar radius falls along straight lines in the temperature-luminosity plot (per Equation 1), and surface gravity falls along straight lines in the mass-radius plot (per Equation 2).

The clouds of blue and red dots in Figure 9 are the results of the Monte Carlo calculations, showing the observational constraints on possible primary (blue) and secondary (red) parameters assuming a uniform prior on $\log \left(M_{1}\right)$. The black diamonds are the result: the pa- rameter pair that best-matches with stellar evolutionary tracks simultaneously in mass, radius, temperature, and luminosity.

The colored curves in Figure 9 are selected isochrones from the two sets of stellar evolutionary models. The upper set of isochrones come from the Padova $[\mathrm{M} / \mathrm{H}]=-1$ tracks, stepping from 0.1 Gyr (magenta) to 12 Gyr (red) in factors of 3 in age. The lower set of isochrones come from the Althaus et al. (2013) WD tracks, stepping from 0.15 Gyr (magenta) to 2.4 Gyr (red) in factors of 2 in age. Because Althaus et al. (2013) start their tracks at the moment the WD progenitor detaches from the common envelope phase, the WDs begin hot and extended before cooling and shrinking with age. Note that the selected isochrones in Figure 9 are for display purposes; we search every time step of every track in our comparison.

We find that the observations of $\mathrm{J} 0750+40$ and J0803+41, sdA stars found by Kepler et al., are best explained by the metal-poor main sequence tracks: $\simeq 1.2 M_{\odot}$ stars with $\simeq 0.8 M_{\odot}$ companions. Solutions involving WDs yield 25 times larger residuals. We note that the best-matching surface gravity for $\mathrm{J} 0750+40$, $\log g=4.3$, is in perfect agreement with its SSPP measurement. If we were to use its SSPP effective temperature instead of our pure hydrogen model-derived $T_{\text {eff }}$, the best solution to $\mathrm{J} 0750+40$ shifts slightly to a 1.15 $M_{\odot}$ and $0.7 M_{\odot}$ pair of metal-poor main sequence stars.

$\mathrm{J} 1600+27$, one of our sdA-like ELM WD candidates, is ambiguous. Formally, the best solution is a $0.16 M_{\odot}$ helium-core $\mathrm{WD}$ orbiting a $\simeq 0.8 M_{\odot}$ main sequence companion. J1600+27's light curve fit yields a companion star that is more luminous and larger in radius than the hot "primary," which means the hot primary could be a WD. However, this would indicate that we caught $\mathrm{J} 1600+27$ right after the WD progenitor detached from the common envelope phase, which seems unlikely. Parameter solutions involving metal poor 0.95 $M_{\odot}+0.95 M_{\odot}$ main sequence stars are also possible with only 3 times larger residuals, in better agreement with J1600+27's SED. Higher resolution spectroscopy would resolve the issue, as an equal mass system would be a double-lined spectroscopic binary.

Two other eclipsing sdA stars that have infrared excess are J0802+43 and J0832+13. Neither system shows significant radial velocity variability. Instead, we perform our Monte Carlo calculation using our radial velocities as upper limits on the velocity amplitudes. We find that the observations are best explained by main sequence tracks: $\simeq 1.9 M_{\odot}$ stars with $\simeq 1.0 M_{\odot}$ companions if solar metallicity, or $\simeq 1.3 M_{\odot}$ stars with $\simeq 0.8$ $M_{\odot}$ companions if metal-poor. The upper limits on velocity push the solutions towards more massive stars, 

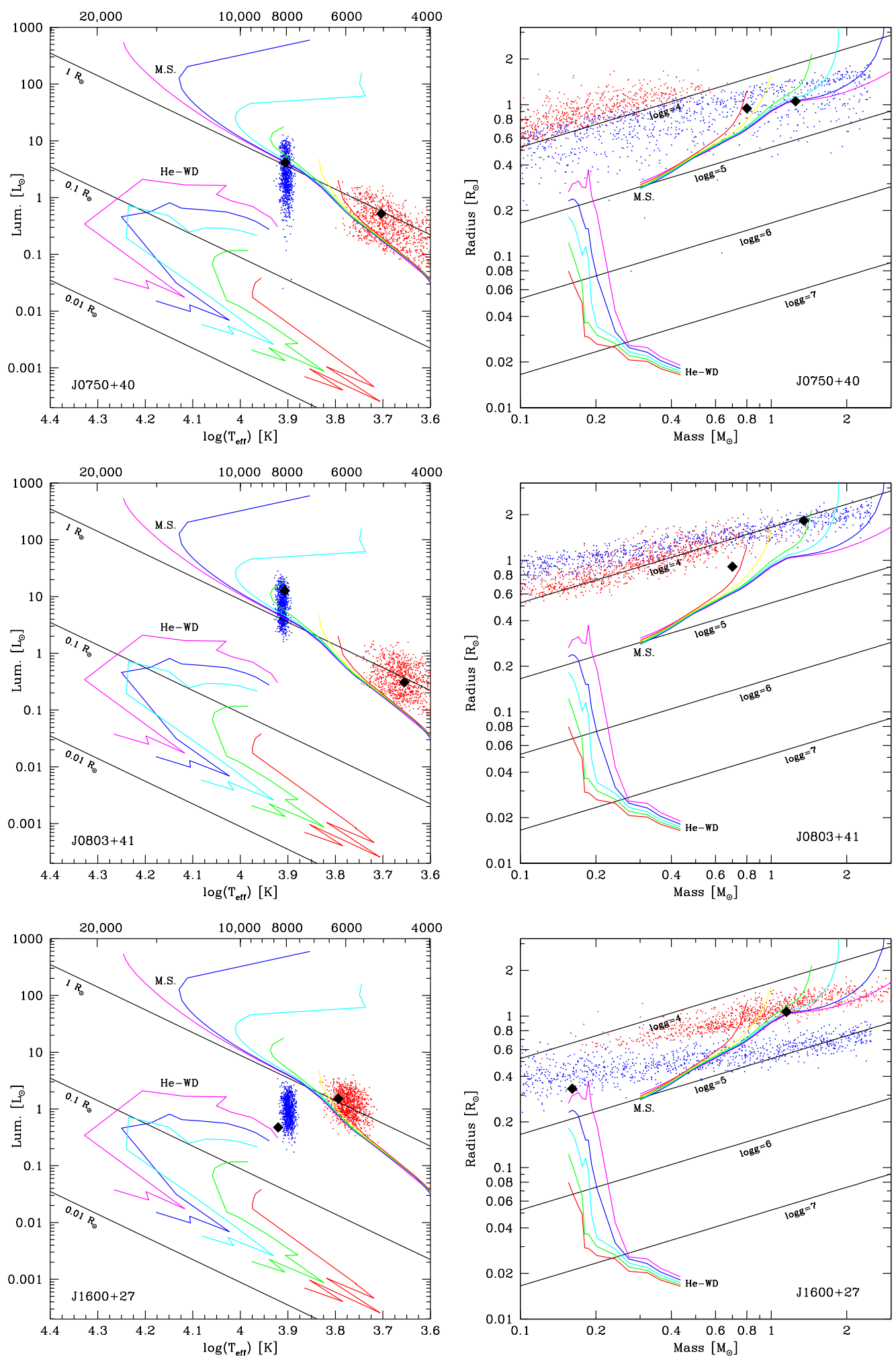

Figure 9. Temperature-luminosity (left) and mass-radius (right) diagrams showing the joint constraints on J0750+40 (top), J0803+41 (middle), and J1600+27 (bottom). Possible primary parameters (blue dots) and secondary parameters (red dots) are shown for a uniform prior on $M_{1}$. Colored curves are selected isochrones from Padova $[\mathrm{M} / \mathrm{H}]=-1$ tracks ranging from 0.1 Gyr to 12 Gyr (upper right) and from Althaus et al. (2013) helium-core WD tracks ranging from 0.1 Gyr to 2.4 Gyr (lower left). Black diamonds are the result: the primary and secondary parameter pair that most closely matches the full set of tracks simultaneously in mass, radius, temperature, and luminosity. 
and solar metallicity stars are more massive than metalpoor stars of identical temperature. However SSPP reports $[\mathrm{Fe} / \mathrm{H}]=-0.971 \pm 0.012$ for $\mathrm{J} 0802+43$ (see Table 2) which clearly points to the metal-poor solution. Parameter solutions involving WDs have 1000 times larger residuals than main sequence star solutions.

In summary, we consider the joint constraints on five objects with eclipses, radial velocity orbits, and/or infrared excess. The four objects identified as sdA stars by Kepler are metal-poor $\simeq 1.2 M_{\odot}$ stars with $\simeq 0.8 M_{\odot}$ companions; the object selected as an ELM WD candidate is either a low mass WD with a main sequence companion or an equal-mass main sequence binary.

\subsection{Comparison with Other Results}

The photometric variability study of Bell et al. (2017) provides an interesting comparison. They target nine $\sim 8000 \mathrm{~K}$ ELM WD candidates suspected to fall in the empirical WD instability strip. Three objects pulsate: two may be ELM WD pulsators; one is clearly a highamplitude $\delta$ Scuti variable. Two other objects are photometric binaries: one has ellipsoidal variations consistent with an ELM WD binary; the other has $\simeq 4 \mathrm{hr}$ eclipses and must be a main sequence star. They conclude that ELM Survey objects with sdA temperatures are a mix of WDs and main sequence stars.

It is also interesting to check the kinematics of the five eclipsing binaries studied here. An $8000 \mathrm{~K}$ metal-poor main sequence star with absolute magnitude $M_{g} \simeq 2.5$ is a 1000 times more luminous than an $8000 \mathrm{~K}$ ELM WD with $M_{g} \simeq 10$ (Bressan et al. 2012; Althaus et al. 2013). The objects studied here have a typical apparent magnitude of $g=18$. Thus any metal-poor $1.2 M_{\odot}$ star would be about $13 \mathrm{kpc}$ distant in the halo, while a ELM WD would be about $0.4 \mathrm{kpc}$ distant and in the disk.

Systemic radial velocities for the eclipsing binaries $\mathrm{J} 0832+13$ and $\mathrm{J} 1600+27$ are $+135 \mathrm{~km} \mathrm{~s}^{-1}$ and -294 $\mathrm{km} \mathrm{s}^{-1}$, respectively, consistent with halo stars. Systemic radial velocities for the other three eclipsing binaries are around $50 \mathrm{~km} \mathrm{~s}^{-1}$, and thus ambiguous. Proper motions for the five eclipsing binaries are all about $3 \pm 3$ mas $\mathrm{yr}^{-1}$, consistent with zero (Altmann et al. 2017). Small proper motions are expected for distant stars, however the large inferred $\sim 200 \mathrm{~km} \mathrm{~s}^{-1}$ tangential velocities are statistically insignificant given the errors. Thus the kinematics are consistent with halo stars, but not a significant constraint.

Taking this argument in the other direction, an sdA star with a high proper motion may be the signature of a nearby ELM WD. There are 1,364 sdA stars in the full Kepler et al. (2015, 2016) sample detected in at least 6 epochs in the HSOY proper motion catalog;
323 have non-zero proper motions at $>3-\sigma$ confidence (Altmann et al. 2017). If we assume a fiducial absolute magnitude $M_{g}=2.5$ appropriate for a metal-poor $8000 \mathrm{~K}$ main sequence star, then 35 (or $3 \%$ ) of the putative halo sdA stars are unbound to the Milky Way at $>3-\sigma$ confidence. We consider unbound tangential velocities implausible given that the stars all have modest radial velocities. It is much more likely that these 35 sdA stars are WDs with $M_{g} \simeq 10$, in which case their tangential velocities are comparable to their radial velocities. Including distance errors would reduce the number of significant tangential velocity outliers, but, on the basis of proper motions, we conclude that a few percent of the sdA objects may be WDs.

\section{DISCUSSION}

The eclipsing sdA binaries prove one thing: the sdA population contains metal-poor main sequence stars. ELM WDs that have cooled to A-F type temperatures, objects like the eclipsing system NLTT 11748 (Steinfadt et al. 2010; Kawka et al. 2010; Kilic et al. 2010), are presumably mixed into the sdA population. But what fraction of sdA stars are metal-poor main sequence stars, and what fraction are ELM WDs? The eclipsing binaries cannot answer that question, because we do not know if the binaries fairly sample the sdA population. Instead, we turn to theoretical evolutionary tracks and the magnitude-limited ELM WD sample.

Evolutionary tracks provide a precise constraint on the time an ELM WD spends at sdA-like temperatures. The accuracy is complicated by thermonuclear hydrogen shell flashes that alter ELM WD evolutionary times (Driebe et al. 1998; Althaus et al. 2001). The number of shell flashes and the mass threshold at which they occur depend on assumptions about element diffusion, progenitor metallicity, and rotation (Althaus et al. 2015; Istrate et al. 2016). We consider the models of Althaus et al. (2013) and the models of Istrate et al. (2016) with rotation. These two sets of models agree to within $15 \%$ on ELM WD mass and luminosity at a given $T_{\text {eff }}$ and $\log g$, however the time to reach that temperature can in some cases vary by more than a factor of 2 .

What is true across all models is that a magnitudelimited sample contains fewer cool ELM WDs, because cooler WDs are less luminous than hotter WDs. Furthermore, a fraction of ELM WDs must merge with their companions before they cool to $\sim 8000 \mathrm{~K}$ temperatures. We account for mergers by assuming that ELM WDs have formed continuously over the past Gyr with the orbital period distribution derived by Brown et al. (2016b). Following the Brown et al. (2016b) approach 
to evolving the ELM WD population, the ratio of $6500<$ $T_{\text {eff }}<9000 \mathrm{~K}$ to $10,000<T_{\text {eff }}<15,000 \mathrm{~K}$ ELM WDs in a magnitude-limited sample is about 1:2 averaged over the two sets of evolutionary models.

The ELM Survey has now obtained a spectrum for every star in SDSS in the magnitude range $15<g<20$ within the ELM Survey color cut. While some ELM WDs must fall outside the color cut, the ELM Survey provides a robust lower limit on the absolute number of ELM WDs. The clean sample of ELM WD binaries contains 36 objects with $10,000<T_{\text {eff }}<15,000 \mathrm{~K}$. Assuming that the true absolute number of ELM WDs in the SDSS footprint is a few times larger than the published number, the evolutionary model scaling predicts about 50 ELM WDs with $6500<T_{\text {eff }}<9000 \mathrm{~K}$ in the magnitude range $15<g<20$ in the SDSS footprint.

By comparison, Kepler et al. (2015, 2016) identify about 2600 sdA stars with $6500<T_{\text {eff }}<9000 \mathrm{~K}$ and $5<\log g<6.5$ in SDSS. The sdA spectroscopic sample is incomplete. Thus cool ELM WDs comprise no more than about $2 \%$ of the sdA population. This estimate is consistent with the number of tangential velocity outliers in the sdA sample, a completely independent constraint. We conclude that most sdA stars, based on the observations presented here, are likely metal-poor A- and F-type main sequence stars in the halo.

\subsection{Implications}

The physical nature of sdA stars has important implications for their binary properties. The minimum orbital period for a detached binary is set by the Roche lobe radius (Eggleton 1983). If we adopt $q=0.67$ seen in the eclipsing binaries, and assume that all sdA stars are $\simeq 1.2 M_{\odot}$ metal-poor stars, then the Roche lobe criterion demands that no sdA binary has $P<9 \mathrm{hr}$.

Indeed, no $P<9$ hr system has yet been found among the sdA stars. Finding a $P<9$ hr system would be evidence that the binary contains a WD. If sdA stars were solar metallicity stars, on the other hand, the period minimum is about $24 \mathrm{hr}$. This is already ruled out by J0421+00, J0823+37, and possibly J1541+31.

Two-thirds of ELM WDs are observed in detached binaries with orbital periods $P<9 \mathrm{hr}$ (Brown et al. 2016 b), binaries that cannot fit sdA stars. The implication is clear: the Roche lobe criterion sets a minimum binary orbital period for sdA stars 50 times larger than that of ELM WDs.

The physical nature of sdA stars also has implications for studies of pulsating ELM WDs. First discovered by Hermes et al. (2012b, 2013b), the first three ELM WD pulsators are hotter than $9000 \mathrm{~K}$ and are in binary systems with $P=4.1 \mathrm{hr}$ to $14.6 \mathrm{hr}$. The pulsat- ing ELM WD companion to the millisecond pulsar PSR J1738+0333 has similar properties (Kilic et al. 2015). The temperatures and orbital periods of these pulsators are consistent with being ELM WDs.

However, the fourth and fifth members of this class of pulsators are cooler than $9000 \mathrm{~K}$ and do not show any significant radial velocity variations (Hermes et al. 2013a). Corti et al. (2016) and Bell et al. (2017) present five additional pulsators with temperatures below $8000 \mathrm{~K}$, and with no evidence of binarity. Because pulsations are common in A-type stars, it is possible that these relatively cool pulsators are SX Phe variables, and not pulsating ELM WDs. Hence, there may be only four likely pulsating ELM WDs: the three pulsators presented in Hermes et al. (2013b) and the WD companion to PSR J1738+0333 (Kilic et al. 2015). Measuring the rate of pulsation period change could clarify their stellar nature, because different classes of pulsators have different rates of period change (Calcaferro et al. 2017).

\section{SUMMARY}

In this paper we investigate the physical nature of $\operatorname{sdA}$ stars and their possible link to ELM WDs. The distribution of colors and reduced proper motions indicate that sdA stars are cooler and more luminous, and thus larger in radius, than published ELM WDs. We perform a detailed study of sdA stars in eclipsing binaries with infrared excess and/or radial velocity orbital solutions. The joint observational constraints are best explained with binaries containing metal-poor $\simeq 1.2 M_{\odot}$ main sequence stars with $\simeq 0.8 M_{\odot}$ companions, not with $\mathrm{ELM}$ WDs.

The source of confusion comes from fitting pure hydrogen models to sdA spectra. Metal line blanketing is important below $9000 \mathrm{~K}$, and the Balmer lines become insensitive to temperature (Strömgren 1969). We demonstrate that pure hydrogen model fits to synthetic A-F type spectra yield systematically wrong surface gravities by $\sim 1$ dex. Empirically, surface gravities derived by SSPP for a set of sdA stars differ by 1.8 dex compared to their pure hydrogen model derived values. Thus sdA stars fit with pure hydrogen models appear to be WD imposters.

While it is true that ELM WDs must exist at sdAlike temperatures, they are intrinsically faint and sufficiently rare that we predict about 50 cool ELM WDs with $15<g<20 \mathrm{mag}$ in the SDSS survey. Thus ELM WDs comprise of order $1 \%$ of the observed sdA population. This conclusion is supported by the small number of sdA tangential velocity outliers, and the absence of $P<9$ hr periods in observed sdA binaries. The major- 
Table 5. Radial Velocity Data

\begin{tabular}{ccc}
\hline \hline Object & HJD & $\begin{array}{c}v_{\text {helio }} \\
\left(\mathrm{km} \mathrm{s}^{-1}\right)\end{array}$ \\
\hline J0747+45 & 7336.949516 & $64.11 \pm 5.20$ \\
$\ldots$ & 7337.022786 & $60.35 \pm 5.05$ \\
$\ldots$ & 7427.696022 & $84.46 \pm 8.43$ \\
$\ldots$ & 7427.859036 & $87.48 \pm 8.18$ \\
$\ldots$ & 7428.695398 & $99.08 \pm 7.05$ \\
$\ldots$ & 7430.691555 & $73.15 \pm 7.84$ \\
J0750+40 & 7336.962809 & $-20.97 \pm 4.66$ \\
$\ldots$ & 7337.017686 & $-37.19 \pm 7.11$ \\
$\ldots$ & 7427.717586 & $-12.25 \pm 6.72$ \\
$\ldots$ & 7427.852464 & $-31.56 \pm 7.02$ \\
\hline
\end{tabular}

Note-This table is available in its entirety in machine-readable and Virtual Observatory forms in the online journal. A portion is shown here for guidance regarding its form and content.

ity of sdA stars are likely metal-poor A-F type stars in the halo.

We thank S. O. Kepler for providing us with the list of sdA eclipsing binary candidates. We thank B. Kunk, E. Martin, and A. Milone for their assistance with observations obtained at the MMT Observatory, P. Canton for his assistance with observations obtained at Kitt Peak National Observatory, and P. Berlind and M. Calkins for their assistance with observations obtained at the Fred Lawrence Whipple Observatory. MK and AG gratefully acknowledge the support of the NSF and NASA under grants AST-1312678 and NNX14AF65G. This project makes use of data products from the Sloan
Digital Sky Survey, which is managed by the Astrophysical Research Consortium for the Participating Institutions. This project makes use of data products from the Catalina Sky Surveys. The CSS survey is funded by the National Aeronautics and Space Administration under Grant No. NNG05GF22G issued through the Science Mission Directorate Near-Earth Objects Observations Program. The CRTS survey is supported by the U.S. National Science Foundation under grants AST0909182 and AST-1313422. This research makes use the SAO/NASA Astrophysics Data System Bibliographic Service. This work was supported in part by the Smithsonian Institution.

Facilities: MMT (Blue Channel Spectrograph), Mayall (KOSMOS), FLWO:1.5m (FAST)

\section{APPENDIX}

\section{A. DATA TABLE}

Table 5 presents the radial velocity measurements for the sample of 11 sdA stars suspected of being eclipsing binaries and 11 ELM WD candidates that have sdA-like temperatures. Table 5 columns include object name, heliocentric Julian date (based on UTC), heliocentric radial velocity (uncorrected for the WD gravitational redshift), and velocity error. 


\section{REFERENCES}

Abt, H. A., Levato, H., \& Grosso, M. 2002, ApJ, 573, 359

Abt, H. A., \& Morrell, N. I. 1995, ApJS, 99, 135

Allende Prieto, C., Fernández-Alvar, E., Schlesinger, K. J., et al. 2014, A\&A, 568, A7

Allende Prieto, C., Sivarani, T., Beers, T. C., et al. 2008, AJ, 136, 2070

Althaus, L. G., Camisassa, M. E., Miller Bertolami, M. M., Córsico, A. H., \& García-Berro, E. 2015, A\&A, 576, A9

Althaus, L. G., Miller Bertolami, M. M., \& Córsico, A. H. 2013, A\&A, 557, A19

Althaus, L. G., Serenelli, A. M., \& Benvenuto, O. G. 2001, MNRAS, 324, 617

Altmann, M., Roeser, S., Demleitner, M., Bastian, U., \& Schilbach, E. 2017, A\&A, accepted

Bell, K. J., Gianninas, A., Hermes, J. J., et al. 2017, ApJ, submitted

Breger, M. 2000, in ASP Conf. Ser. Vol. 210, Delta Scuti and Related Stars, ed. M. Breger \& M. Montgomery (San Francisco: ASP), 3

Bressan, A., Marigo, P., Girardi, L., et al. 2012, MNRAS, 427, 127

Brown, W. R., Gianninas, A., Kilic, M., Kenyon, S. J., \& Allende Prieto, C. 2016a, ApJ, 818, 155

Brown, W. R., Kilic, M., Allende Prieto, C., Gianninas, A., \& Kenyon, S. J. 2013, ApJ, 769, 66

Brown, W. R., Kilic, M., Allende Prieto, C., \& Kenyon, S. J. 2010, ApJ, 723, 1072

-. 2012, ApJ, 744, 142

Brown, W. R., Kilic, M., Hermes, J. J., et al. 2011, ApJL, 737, L23

Brown, W. R., Kilic, M., Kenyon, S. J., \& Gianninas, A. 2016b, ApJ, 824, 46

Calcaferro, L. M., Córsico, A. H., \& Althaus, L. G. 2017, A\&A, accepted

Carter, J. A., Rappaport, S., \& Fabrycky, D. 2011, ApJ, 728,139

Castelli, F., Gratton, R. G., \& Kurucz, R. L. 1997, A\&A, 318,841

Castelli, F., \& Kurucz, R. L. 2004, arXiv:astro-ph/0405087

Claret, A., \& Bloemen, S. 2011, A\&A, 529, A75

Corti, M. A., Kanaan, A., Córsico, A. H., et al. 2016, A\&A, 587, L5

Drake, A. J., Djorgovski, S. G., Mahabal, A., et al. 2009, ApJ, 696, 870

Driebe, T., Schoenberner, D., Bloecker, T., \& Herwig, F. 1998, A\&A, 339, 123

Eggleton, P. P. 1983, ApJ, 268, 368

Fabricant, D., Cheimets, P., Caldwell, N., \& Geary, J. 1998, PASP, 110, 79
Gentile Fusillo, N. P., Gänsicke, B. T., \& Greiss, S. 2015, MNRAS, 448, 2260

Gianninas, A., Bergeron, P., \& Ruiz, M. T. 2011, ApJ, 743, 138

Gianninas, A., Dufour, P., \& Bergeron, P. 2004, ApJL, 617, L57

Gianninas, A., Dufour, P., Kilic, M., et al. 2014a, ApJ, 794, 35

Gianninas, A., Hermes, J. J., Brown, W. R., et al. 2014b, ApJ, 781, 104

Gianninas, A., Kilic, M., Brown, W. R., Canton, P., \& Kenyon, S. J. 2015, ApJ, 812, 167

Hermes, J. J., Brown, W. R., Kilic, M., et al. 2014, ApJ, 792,39

Hermes, J. J., Gaensicke, B. T., \& Breedt, E. 2017, in ASP Conf. Ser.: 20th European Workshop on White Dwarfs, ed. P.-E. Tremblay, B. Gaensicke, \& T. Marsh (San Francisco: ASP), in press

Hermes, J. J., Kilic, M., Brown, W. R., et al. 2012a, ApJL, $757, \mathrm{~L} 21$

Hermes, J. J., Montgomery, M. H., Gianninas, A., et al. 2013a, MNRAS, 436, 3573

Hermes, J. J., Montgomery, M. H., Winget, D. E., et al. 2012b, ApJL, 750, L28

—. 2013b, ApJ, 765, 102

Iben, Jr., I. 1990, ApJ, 353, 215

Istrate, A. G., Marchant, P., Tauris, T. M., et al. 2016, A\&A, 595, A35

Kawka, A., Vennes, S., \& Vaccaro, T. R. 2010, A\&A, 516, L7

Kenyon, S. J., \& Garcia, M. R. 1986, AJ, 91, 125

Kepler, S. O., Pelisoli, I., Koester, D., et al. 2015, MNRAS, 446, 4078

—. 2016, MNRAS, 455, 3413

Kilic, M., Brown, W. R., Allende Prieto, C., Kenyon, S. J., \& Panei, J. A. 2010, ApJ, 716, 122

Kilic, M., Brown, W. R., Allende Prieto, C., et al. 2011, ApJ, 727, 3

—. 2012, ApJ, 751, 141

Kilic, M., Brown, W. R., Gianninas, A., et al. 2014, MNRAS, 444, L1

Kilic, M., Hermes, J. J., Gianninas, A., \& Brown, W. R. 2015, MNRAS, 446, L26

Kilic, M., Thorstensen, J. R., \& Koester, D. 2008, ApJL, $689, \mathrm{~L} 45$

Kilic, M., et al. 2006, AJ, 131, 582

Kurtz, M. J., \& Mink, D. J. 1998, PASP, 110, 934

Lawrence, A., Warren, S. J., Almaini, O., et al. 2007, MNRAS, 379, 1599 
Lee, Y. S., Beers, T. C., Sivarani, T., et al. 2008, AJ, 136, 2022

Marsh, T. R., Dhillon, V. S., \& Duck, S. R. 1995, MNRAS, 275,828

Martini, P., Elias, J., Points, S., et al. 2014, in Proc. SPIE 9147, Ground-based and Airborne Instrumentation for Astronomy V, ed. Ramsay, S. K and McLean, I. S. and Takami, H. (Montreal, Canada: SPIE), $91470 Z$

Maxted, P. F. L., Bloemen, S., Heber, U., et al. 2014, MNRAS, 437, 1681

Maxted, P. F. L., Serenelli, A. M., Miglio, A., et al. 2013, Nature, 498, 463

North, P., \& Zahn, J.-P. 2004, NewAR, 48, 741

Payne, C. H. 1925, PhD thesis, Harvard University

Pelisoli, I., Kepler, S. O., Koester, D., \& Romero, A. D. 2017, in ASP Conf. Ser.: 20th European Workshop on White Dwarfs, ed. P.-E. Tremblay, B. Gaensicke, \& T. Marsh (San Francisco: ASP), in press

Pickles, A. J. 1998, PASP, 110, 863

Press, W. H., Teukolsky, S. A., Vetterling, W. T., \& Flannery, B. P. 1992, Numerical recipes in C. The art of scientific computing (Cambridge: University Press, 2nd ed.)

Rappaport, S., Nelson, L., Levine, A., et al. 2015, ApJ, 803, 82
Schmidt, G. D., Weymann, R. J., \& Foltz, C. B. 1989, PASP, 101, 713

Skrutskie, M. F., Cutri, R. M., Stiening, R., et al. 2006, AJ, 131,1163

Smolinski, J. P., Lee, Y. S., Beers, T. C., et al. 2011, AJ, 141,89

Southworth, J., Maxted, P. F. L., \& Smalley, B. 2004, MNRAS, 351, 1277

Steinfadt, J. D. R., Kaplan, D. L., Shporer, A., Bildsten, L., \& Howell, S. B. 2010, ApJL, 716, L146

Strom, S. E. 1969, in Theory and Observation of Normal Stellar Atmospheres, ed. O. Gingerich (Cambridge: MIT), 99

Strömgren, B. 1969, in Theory and Observation of Normal Stellar Atmospheres, ed. O. Gingerich (Cambridge: MIT), 337

Strömgren, B., Gyldenkaerne, K., Rudkjobing, M., \& Thernoee, K. A. 1944, Publikationer og mindre Meddeler fra Kobenhavns Observatorium, 138, 1

Tremblay, P.-E., Gianninas, A., Kilic, M., et al. 2015, ApJ, 809,148

Tremblay, P.-E., Ludwig, H.-G., Steffen, M., \& Freytag, B. 2013, A\&A, 559, A104

Vennes, S., Thorstensen, J. R., Kawka, A., et al. 2011, ApJL, 737, L16

Webbink, R. F. 1984, ApJ, 277, 355

Zuckerman, B., Koester, D., Melis, C., Hansen, B. M., \& Jura, M. 2007, ApJ, 671, 872 